\title{
CHARACTERIZATION OF SOME ZERO-DIMENSIONAL SEPARABLE METRIC SPACES
}

BY

JAN VAN MILL

\begin{abstract}
Let $X$ be a separable metric zero-dimensional space for which all nonempty clopen subsets are homeomorphic. We show that, up to homeomorphism, there is at most one space $Y$ which can be written as an increasing union $\bigcup_{n=1}^{\infty} F_{n}$ of closed sets so that for all $n \in \mathbf{N}, F_{n}$ is a copy of $X$ which is nowhere dense in $F_{n+1}$. If moreover $X$ contains a closed nowhere dense copy of itself, then $\boldsymbol{Y}$ is homeomorphic to $\mathbf{Q} \times X$ where $\mathbf{Q}$ denotes the space of rational numbers. This gives us topological characterizations of spaces such as $\mathbf{Q} \times \mathbf{C}$ and $\mathbf{Q} \times \mathbf{P}$.
\end{abstract}

1. Introduction. All topological spaces under discussion are separable metric.

It is well known that there are good usable topological characterizations of the space of rational numbers $\mathbf{Q}$, the space of irrational numbers $\mathbf{P}$ and of the Cantor set $\mathbf{C}$ (see, respectively, [15], [1] and [3]). Less well known is that there is also a characterization of $\mathbf{Q} \times \mathbf{C}[1]$. Since $\mathbf{Q} \times \mathbf{Q} \approx \mathbf{Q}, \mathbf{P} \times \mathbf{P} \approx \mathbf{P}, \mathbf{C} \times \mathbf{C} \approx \mathbf{C}$ and $\mathbf{P} \times \mathbf{C} \approx \mathbf{P}$ the question naturally arises whether there is a (nontrivial) characterization of $\mathbf{Q} \times \mathbf{P}$ (this was brought to my attention by Eric van Douwen). The aim of this paper is to give such a characterization. We will prove that $\mathbf{Q} \times \mathbf{P}$ is (topologically) the unique space which can be written as an increasing union $\cup_{n=1}^{\infty} F_{n}$ of closed sets so that, for all $n \in \mathbf{N}, F_{n}$ is a copy of $\mathbf{P}$ which is nowhere dense in $F_{n+1}$. The characterizations of $\mathbf{Q}, \mathbf{P}, \mathbf{C}$ and $\mathbf{Q} \times \mathbf{C}$ are of a different nature than our characterization of $\mathbf{Q} \times \mathbf{P}$ and one wonders whether there is a more "classical" characterization. By a classical characterization of a space $X$ we mean a statement such as: $Y$ is homeomorphic to $X$ iff $Y$ has topological property $\mathscr{P}_{0}$ but no nonempty open subset of $Y$ has topological property $\mathscr{P}_{1}$. For example, $Y \approx Q$ iff $Y$ is countable and no nonempty open subspace of $Y$ is compact, or, what is the same, $\mathbf{Q}$ is the unique countable nowhere locally compact space [15]. Using the above characterization of $\mathbf{Q} \times \mathbf{P}$ we will show that $\mathbf{Q} \times \mathbf{P}$ is the unique zero-dimensional space $X$ with the following properties:

(a) $X$ is the union of countably many closed and topologically complete subspaces;

(b) $X$ is nowhere complete and nowhere $\sigma$-compact.

Analyzing our original proof of the first characterization it occurred to us that we "only" used that $\mathbf{P}$ is a zero-dimensional space with the pleasant property that all nonempty clopen subspaces are homeomorphic and that $\mathbf{P}$ contains a nowhere dense closed copy of itself. Let us call a space strongly homogeneous provided that

Received by the editors January 29, 1980.

1980 Mathematics Subject Classification. Primary 54E45, 54E50, 54D35.

(C) 1981 American Mathematical Society 0002-9947/81/0000-0114/\$03.75 
all nonempty clopen subspaces are homeomorphic. Notice that each connected space is strongly homogeneous so that in general strong homogeneity has nothing to do with homogeneity. However, since in this paper we only work with zero-dimensional spaces and since clearly each zero-dimensional strongly homogeneous space is homogeneous, in the realm of zero-dimensional spaces this justifies the terminology. Our basic tool is that strongly homogeneous zero-dimensional spaces satisfy a very strong homogeneity property, namely that each homeomorphism between closed and nowhere dense subsets can be extended to an autohomeomorphism of the whole space. We say that strongly homogeneous zero-dimensional spaces satisfy the Homeomorphism Extension Theorem (this term was first used in infinite-dimensional topology). We also say that if $X$ is a space, the space $Y \in$ $\operatorname{CAP}(X)$ provided that $Y$ can be written as an increasing union $\cup_{n=1}^{\infty} F_{n}$ of closed sets so that, for each $n \in \mathrm{N}, F_{n}$ is a copy of $X$ which is nowhere dense in $F_{n+1}$. This definition is motivated by R. D. Anderson's [2] notion of a capset for the Hilbert cube. Using the Homeomorphism Extension Theorem we will show, by a conceptually simple but unpleasant construction, that if $X$ is a strongly homogeneous zero-dimensional space, up to homeomorphism, $\operatorname{CAP}(X)$ contains at most one space. Using again the Homeomorphism Extension Theorem we prove that if $X$ contains a closed nowhere dense copy of itself then $\mathbf{Q} \times X \in \operatorname{CAP}(X)$, thus establishing a topological characterization of $\mathbf{Q} \times X$.

The results in this paper suggest many questions. At several places we use that our spaces are strongly homogeneous. The first question which arises is whether strong homogeneity can be replaced by homogeneity. The answer to this question is in the negative. Eric van Douwen [4] has recently obtained the beautiful result that there is a zero-dimensional topological group $H$ with (among others) the following property: if $F$ and $G$ are homeomorphic closed subspaces of $H$ then $H-F$ and $H-G$ are homeomorphic (notice that we do not require that $F$ and $G$ are proper closed subspaces of $H$ ). This implies that $H$ is not strongly homogeneous and that $H$ does not contain a nowhere dense closed copy of itself. It is likely that $H$ satisfies the Homeomorphism Extension Theorem, or, if not (which would be highly interesting), that a similar space can be constructed which does satisfy it. It follows that $\operatorname{CAP}(H)=\varnothing$ and our methods do not apply to get a characterization of $\mathbf{Q} \times H$.

1.1. Question. Is there a zero-dimensional topological group $S$ containing two nowhere dense Cantor sets $F$ and $G$ whose complements are not homeomorphic?

The second question which arises is whether strong homogeneity of a zero-dimensional space $X$ suffices to ensure that $\operatorname{CAP}(X)$ is nonempty. In an earlier version of this paper I asked whether there is a strongly homogeneous zero-dimensional space which is not homeomorphic to any nowhere dense closed subspace. This question was answered in the affirmative recently by Eric van Douwen [5].

Throughout this paper some knowledge of Boolean algebras is assumed. All necessary information can be found in Halmos [10].

I am indebted to R. D. Anderson and to Eric van Douwen for some helpful comments during the preparation of this paper. 
2. Notation and some preliminary lemmas. A clopen set refers to a set which is both closed and open, and by a clopen cover of a space we mean a cover consisting of nonempty clopen sets. Also, a cover is called disjoint if its elements are pairwise disjoint. If $\mathcal{Q}$ and $\mathcal{V}$ are both covers of a space then $\mathcal{U}$ refines $\mathcal{V}$ if each $U \in \mathcal{U}$ is contained in some $V \in \mathfrak{V}$. Let $X \subset Y$ and let $\mathscr{U}$ be a cover of $Y$. Then $\mathscr{X} \boldsymbol{Q}=\{U \cap X: U \in \mathcal{U}\}$.

All metrics are assumed to be totally bounded. If $x \in X$ and $\varepsilon>0$ then $U_{e}(x)=$ $\{y \in X: d(x, y)<\varepsilon\}$. The diameter, $\operatorname{diam}(A)$, of a set $A \subset X$ is $\sup \{d(x, y)$ : $x, y \in A\}$. If $\mathscr{U}$ is a collection of subsets of $X$ then mesh $\mathscr{U}=\sup \{\operatorname{diam}(U)$ : $U \in \mathcal{Q}\}$.

If $\mathscr{U}$ is a collection of subsets of a set $X$ and if $f: X \rightarrow Y$ is a function then, for simplicity, we write $f(\mathcal{Q})=\{f[U]: U \in \mathscr{Q}\}$.

2.1. Lemma. Let $X$ be a zero-dimensional space and let $F \subset X$ be a closed subspace. If $U \subset F$ is clopen (in $F$ ) then for each $\varepsilon>0$ there is a clopen $U^{\prime} \subset X$ so that $U^{\prime} \cap F=U$ and $\operatorname{diam}\left(U^{\prime}\right)<\operatorname{diam}(U)+\varepsilon$.

Proof. Since $U$ and $F-U$ are disjoint closed subsets of $X$ there are open disjoint $V_{0}, V_{1} \subset X$ so that $V_{0} \cap F=U$ and $V_{1} \cap F=F-U$. Let $C$ be a clopen neighborhood of $F$ so that $C \cap\left(\left(X-\left(V_{0} \cup V_{1}\right)\right) \cup\left\{y \in X: d(y, F)>\frac{1}{4} \varepsilon\right\}\right)=\varnothing$. Put $U^{\prime}=C \cap V_{0}$. It is clear that $U^{\prime}$ defined in this way is as required.

2.2. Lemma. Let $X$ be a zero-dimensional space and let $f: X \rightarrow Y$ be a homeomorphism. In addition, let $\mathcal{Q}$ be a finite open cover of $X$ and let $\mathcal{V}$ be a finite open cover of $Y$. Then for each $\varepsilon>0$ there is a finite disjoint clopen cover $\mathcal{Q}$ of $X$ so that

(i) W refines $थ$ and mesh $थ \leqslant \varepsilon$;

(ii) $f(\mathscr{W})$ refines $\mathcal{V}$ and $\operatorname{mesh} f(\mathscr{W})<\varepsilon$.

Proof. Without loss of generality assume that mesh $\mathcal{U}<\varepsilon$ and mesh $\mathcal{V}<\varepsilon$. Now let $\mathscr{W}$ be a finite disjoint clopen cover of $X$ both refining $\mathscr{U}$ and $\left\{f^{-1}[V]\right.$ : $V \in \mathfrak{V}\}$.

A compactification of a space $X$ is a compact space $\tilde{X}$ containing $X$ as a dense subspace. The following result is due to de Groot and McDowell [8]. For completeness sake we include an easier proof of this result than the one in [8].

2.3. Lemma. Let $X$ be a zero-dimensional space and let $\Phi: X \rightarrow X$ be an autohomeomorphism. Then there is a zero-dimensional compactification $\tilde{X}$ of $X$ so that $\Phi$ can be extended to an autohomeomorphism $\tilde{\boldsymbol{\Phi}}$ of $\tilde{X}$.

Proof. Let $\mathcal{C}$ be the Boolean algebra of clopen subsets of $X$ and let $\mathscr{B} \subset \mathcal{C}$ be a countable subfamily which is a base for $X$. Put $\mathcal{E}=\cup_{n \in Z} \Phi^{n}(\mathscr{B})$ and let $\mathscr{F}$ be the smallest Boolean subalgebra of $\mathcal{C}$ containing $\mathcal{E}$. Notice that $\mathscr{F}$ is countable and that the function $\Phi^{\#}: \mathcal{F} \rightarrow \mathscr{F}$ defined by $\Phi^{\#}(F)=\Phi[F]$ is a Boolean isomorphism. Let $\tilde{X}$ be the Stone space of $\mathscr{F}$ (identify $X$ and $\{\mathcal{G} \in \tilde{X}: \cap \mathcal{G} \neq \varnothing\}$ ). 
2.4. Lemma. Let $X$ be a zero-dimensional space, let $F$ be a closed subspace of $X$ and let $\tilde{F}$ be a zero-dimensional compactification of $F$. Then there is a zero-dimensional compactification $\tilde{X}$ of $X$ so that the closure $\bar{F}$ of $F$ in $\tilde{X}$ is homeomorphic to $\tilde{F}$ by a homeomorphism which leaves $F$ pointwise fixed.

Proof. Let $\mathscr{B}$ be the Boolean algebra of clopen subsets of $\tilde{F}$ (notice that $\mathscr{B}$ is countable) and let $\varepsilon$ be a countable clopen basis for $X$. For each $B \in \mathscr{B}$ and $E \in \mathcal{E}$ for which $B \cap F \subset E$ choose a clopen $C \subset X$ so that $C \subset E$ and $C \cap F=B \cap F$. Let $\mathcal{C}$ denote the collection of clopen subsets of $X$ obtained in this way. Notice that $\mathcal{C}$ is countable. Let $\mathcal{F}$ be the smallest Boolean subalgebra of the Boolean algebra of clopen subsets of $X$ containing both $\mathcal{C}$ and $\{E \in \mathcal{E}$ : $E \subset X-F\}$. Let $\tilde{X}$ be the Stone space of $\mathscr{F}$ (identify $X$ and $\{\mathcal{G} \in \tilde{X}: \cap \mathcal{G} \neq$ $\varnothing\})$. The function $\Phi: \bar{F} \rightarrow \tilde{F}$ defined by

$$
\{\Phi(\mathcal{G})\}=\bigcap_{G \in \mathcal{G}} \mathrm{cl}_{\tilde{F}} G
$$

can easily be seen to be a homeomorphism of $\bar{F}$ onto $\tilde{F}$ which leaves $F$ pointwise fixed.

3. The Homeomorphism Extension Theorem. In this section we will prove the Homeomorphism Extension Theorem for strongly homogeneous zero-dimensional spaces. This improves results of Pollard [14] and Ryll-Nardzewski (see Knaster and Reichbach [12]).

3.1. ThEOREM. Let $X$ be a strongly homogeneous zero-dimensional space and let $F$ and $G$ be closed nowhere dense subsets of $X$. If $f: F \rightarrow G$ is a homeomorphism then $f$ can be extended to an autohomeomorphism $\bar{f}$ of $X$.

Proof. For convenience we consider two disjoint copies $X_{0}$ and $X_{1}$ of $X$ and we assume that $F$ is a nowhere dense closed subset of $X_{0}$ and that $G$ is a nowhere dense closed subset of $X_{1}$. Let $f: F \rightarrow G$ be a homeomorphism. We will prove that there is a homeomorphism $\bar{f}$ of $X_{0}$ onto $X_{1}$ which extends $f$. This clearly suffices to prove the theorem.

If $F=\varnothing$ there is nothing to prove, so assume that $F \neq \varnothing$. By Lemmas 2.3 and 2.4 there are zero-dimensional compactifications $\tilde{X}_{0}$ and $\tilde{X}_{1}$ of, respectively, $X_{0}$ and $X_{1}$ so that $f$ can be extended to a homeomorphism $\tilde{f}: \bar{F} \rightarrow \bar{G}$. Without loss of generality assume that $\tilde{X}_{0} \cap \tilde{X}_{1}=\varnothing$. Consider the topological sum $\tilde{X}_{0}+\tilde{X}_{1}$ and for each $x \in \bar{F}$ identify $x$ and $\tilde{f}(x)$. Call the resulting quotient space $Z$ and let $\pi$ : $\tilde{X}_{0}+\tilde{X}_{1} \rightarrow Z$ be the identification. Let $U=\pi\left[\tilde{X}_{0}-\bar{F}\right], V=\pi\left[\tilde{X}_{1}-\bar{G}\right]$ and $Y=$ $\pi[\bar{F}]$. For each $x \in U$ let $C_{x} \subset Z$ be clopen so that $x \in C_{x} \subset U$ and $\operatorname{diam}\left(C_{x}\right)<$ $d\left(C_{x}, V \cup Y\right)$. There are $x_{n} \in U(n \in \mathbf{N})$ so that $\cup_{n=1}^{\infty} C_{x_{n}}=U$. For each $n \in \mathbf{N}$ put $D_{n}=C_{x_{n}}-\cup_{i=1}^{n-1} C_{x_{i}}$ and without loss of generality assume that all the $D_{n}$ 's are nonempty. Let $\mathscr{D}=\left\{D_{n}: n \in \mathbf{N}\right\}$ and notice that

(a) $\mathscr{D}$ is a disjoint clopen cover of $U$;

(b) each $D \in \mathscr{D}$ is nonempty and $\operatorname{diam}(D)<d(D, V \cup Y)$.

In the same way we can find a disjoint clopen cover $\mathcal{E}=\left\{E_{n}: n \in \mathbf{N}\right\}$ of $V$ so that each $E \in \mathcal{E}$ is nonempty and $\operatorname{diam}(E)<d(E, U \cup Y)$. Notice that $\pi \mid X_{0}-F$ is 
an embedding so that we may identify $X_{0}-F$ and $\pi\left[X_{0}-F\right]$. In the same way, identify $X_{1}-G$ and $\pi\left[X_{1}-G\right]$. Also notice that $X_{0}-F$ is dense in $U$ so that we may pick for each $n \in \mathrm{N}$ a point $p_{n} \in D_{n} \cap\left(X_{0}-F\right)$. In the same way, for each $n \in \mathbf{N}$ pick a point $q_{n} \in E_{n} \cap\left(X_{1}-G\right)$. Since

$$
\left\{p_{n}: n \in \mathbf{N}\right\}^{-}=\left\{p_{n}: n \in \mathbf{N}\right\} \cup Y,
$$

and, similarly,

$$
\left\{q_{n}: n \in \mathbf{N}\right\}^{-}=\left\{q_{n}: n \in \mathbf{N}\right\} \cup Y,
$$

by a result of von Neumann [13, pp. 11-12] (see [11] or [16] for a simpler proof) there is a permutation $\rho$ of $\mathbf{N}$ such that

$$
\lim _{n \rightarrow \infty} d\left(p_{n}, q_{\rho(n)}\right)=0 \text {. }
$$

Since $X$ is strongly homogeneous for each $n \in \mathbf{N}$ there is a homeomorphism $\xi_{n}$ from $D_{n} \cap\left(X_{0}-F\right)$ onto $E_{\rho(n)} \cap\left(X_{1}-G\right)$. Define a function $\Phi:\left(X_{0}-F\right) \cup$ $\pi[F] \rightarrow\left(X_{1}-G\right) \cup \pi[F]$ by

$$
\begin{cases}\Phi(x)=x & (x \in \pi[F]), \\ \Phi(x)=\xi_{n}(x) & \left(x \in D_{n} \cap(X-F)\right) .\end{cases}
$$

We claim that $\Phi$ defined in this way is a homeomorphism. Notice that $\Phi$ is one-to-one and onto.

Fact 1. $\Phi$ is continuous.

Let $x_{n}(n \in \mathbf{N})$ be a sequence in $\left(X_{0}-F\right) \cup \pi[F]$ converging to $x \in\left(X_{0}-F\right)$ $\cup \pi[F]$. It is clear that we only need to consider the case that $x \in \pi[F]$. Without loss of generality $x_{n} \notin \pi[F]$ for all $n \in \mathbf{N}$. For each $n \in \mathbf{N}$ let $k(n) \in \mathbf{N}$ so that $x_{n} \in D_{k(n)}$. Since $\pi[F]$ is dense in $Y, \lim _{n \rightarrow \infty} d\left(x_{n}, Y\right)=0$ and hence, by (a), $\lim _{n \rightarrow \infty} \operatorname{diam}\left(D_{k(n)}\right)=0$ so that the sequence $p_{k(n)}(n \in N)$ converges to $x$. By (*), the sequence $q_{\rho(k(n))}(n \in N)$ converges also to $x$. Since $\lim _{n \rightarrow \infty} \operatorname{diam}\left(E_{\rho(k(n))}\right)=0$ this easily implies that $\Phi\left(x_{n}\right)(n \in N)$ converges to $x(=\Phi(x))$.

Fact 2. $\Phi^{-1}$ is continuous.

It is clear that $\rho^{-1}: \mathbf{N} \rightarrow \mathbf{N}$ is a permutation for which $\lim _{n \rightarrow \infty} d\left(q_{n}, p_{\rho^{-1}(n)}\right)=0$. Applying the same technique as above yields that $\Phi^{-1}$ is continuous.

Hence $\Phi$ is a homeomorphism. Now define $\bar{f}: X_{0} \rightarrow X_{1}$ by

$$
\begin{cases}\bar{f}(x)=f(x) & (x \in F), \\ \bar{f}(x)=\Phi(x) & (x \notin F) .\end{cases}
$$

It is clear that $\bar{f}$ defined in this way is a homeomorphism extending $f$.

3.2. Corollary. Any strongly homogeneous zero-dimensional space is homogeneous.

Notice that in the proof of Theorem 3.1 we have only used that, loosely speaking, $X$ is everywhere locally the same. The same method of proof can therefore be applied to obtain the following result, which will be used in the proof of Theorem 5.1. 
3.3. THEOREM. Let $X$ be a strongly homogeneous zero-dimensional space and let $F$ be a closed nowhere dense subset of $X$. In addition, let $Y$ be a space and let $G \subset Y$ be closed and nowhere dense so that $Y-G$ is homeomorphic to $X$. If $f: F \rightarrow G$ is $a$ homeomorphism then $f$ can be extended to a homeomorphism $\bar{f}: X \rightarrow Y$ (in particular it follows that $X$ and $Y$ are homeomorphic).

3.4. Remark. One might think for a moment that the condition on $Y$ in the above theorem that $Y-G \approx X$ can be weakened to the condition that $Y-G$ is homeomorphic to an open subset of $X$. This is not true however. Let $K_{0}$ and $K_{1}$ be Cantor sets in $\mathbf{C}$ so that $K_{1}$ is nowhere dense in $\mathbf{C}$ and $K_{0}$ is a nowhere dense subset of $K_{1}$. Put $X=\mathrm{C}, F=K_{1}, Y=K_{0} \cup\left(X-K_{1}\right)$ and $G=K_{0}$. It is clear that no homeomorphism $f: K_{1} \rightarrow K_{0}$ can be extended to a homeomorphism $\bar{f}: X \rightarrow Y$.

Let us also notice that although the proofs of Theorems 3.1 and 3.3 are identical the theorems are not equivalent. That the Cantor set satisfies the Homeomorphism Extension Theorem follows from Theorem 3.1 but not from Theorem 3.3.

3.5. Remark. One might ask whether the converse of Theorem 3.1 is true, i.e. whether each zero-dimensional space which satisfies the Homeomorphism Extension Theorem is strongly homogeneous. A discrete space with two points is a counterexample to this question. There is also a counterexample without isolated points. Let $X=\mathrm{C}-\{\mathrm{pt}\}$. Then $X$ is not strongly homogeneous since $X$ has two types of nonempty clopen subsets, namely, the compact ones and the noncompact ones. That $X$ satisfies the Homeomorphism Extension Theorem was proved by Pollard [14]. This also follows from Theorem 3.1. Indeed, let $F$ and $G$ be nowhere dense closed subsets of $X$ and let $f: F \rightarrow G$ be a homeomorphism. If $F$ is compact, then so is $G$, and consequently, there is a compact clopen $C \subset X$ both containing $F$ and $G$. Since $C \approx \mathbf{C}$, Theorem 3.1 implies that $f$ can be extended to a homeomorphism $\tilde{f}: \mathbf{C} \rightarrow \mathbf{C}$. Since $X-C$ is clopen, $\tilde{f}$ can be extended to a homeomorphism $\bar{f}$ : $X \rightarrow X$. If $F$ is not compact then extend $f$ to a homeomorphism $\tilde{f}: F \cup\{\mathrm{pt}\} \rightarrow G$ $\cup\{\mathrm{pt}\}$ by simply sending the point on infinity onto itself. By using Theorem 3.1 the homeomorphism $\tilde{f}$ can be extended to a homeomorphism $\Phi: \mathbf{C} \rightarrow \mathbf{C}$. Now let $\bar{f}=\Phi \mid X$.

4. The main result. In this section we prove our main result that whenever $X$ is a strongly homogeneous zero-dimensional space the set $\operatorname{CAP}(X)$ contains, up to homeomorphism, at most one space. Applications of this result are given in $\$ 5$.

4.1. TheOREM. Let $X$ be a zero-dimensional strongly homogeneous space. Then, up to homeomorphism, $\mathrm{CAP}(X)$ contains at most one space and this space, if it exists, is again strongly homogeneous and zero-dimensional.

Proof. Suppose that $Y_{i}=\cup_{n=1}^{\infty} X_{n}^{i}(i=0,1)$, where, for all $n$,

(i) $X_{n}^{i} \approx X$

(ii) $X_{n}^{i}$ is closed in $Y_{i}$;

(iii) $X_{n}^{i}$ is a nowhere dense subset of $X_{n+1}^{i}$.

First notice that $Y_{i}$ is zero-dimensional being a countable union of zero-dimensional closed subspaces $[6,7.2 .1]$. We will show that $Y_{0} \approx Y_{1}$. 
For each $n \in \mathrm{N}$ we will construct an integer $s_{n}$, an integer $t_{n}$, a finite disjoint clopen cover $\mathscr{Q}_{n}$ of $Y_{0}$, a finite disjoint clopen cover $\mathcal{V}_{n}$ of $Y_{1}$ and a homeomorphism $f_{n}: X_{s_{n}}^{0} \rightarrow X_{t_{n}}^{1}$ so that:

(a) $s_{n-1}<s_{n}$;

(b) $t_{n-1}<t_{n}$;

(c) mesh $\mathscr{Q}_{n} \leqslant 1 / n$ and mesh $\mathscr{V}_{n} \leqslant 1 / n$;

(d) $\mathcal{U}_{n}$ refines $\mathcal{U}_{n-1}$ and $\mathcal{V}_{n}$ refines $\mathcal{V}_{n-1}$;

(e) $X_{s_{n}}^{0}$ meets all elements of $\mathscr{U}_{n}$ and $X_{t_{n}}^{1}$ meets all elements of $\mathfrak{V}_{n}$;

(f) $\forall U \in \mathscr{Q}_{n} \exists V(U) \in \mathscr{V}_{n}: f_{n}\left[U \cap X_{s_{n}}^{0}\right]=V(U) \cap X_{t_{n}}^{1}$;

(g) $\forall U \in \mathscr{Q}_{n} \forall U^{1} \in \mathcal{Q}_{n-1}\left(U \subset U^{1} \rightarrow V(U) \subset V\left(U^{1}\right)\right)$;

(h) $f_{n}$ extends $f_{n-1}$.

Define $s_{1}=1, t_{1}=1$ and let $f_{1}: X_{1}^{0} \rightarrow X_{1}^{1}$ be any homeomorphism. Suppose that we have completed the construction for all $i \leqslant n-1$. Let $\mathcal{Q}^{1}=\mathcal{Q}_{n-1} \mid X_{s_{n-1}}^{0}$ and $\mathcal{V}^{1}=\mathcal{V}_{n-1} \mid X_{t_{n-1}}^{1}$. By Lemma 2.2 there is a finite disjoint clopen cover $W^{1}$ of $X_{s_{n-1}}^{b}$ so that $\mathscr{W}$ refines $\mathcal{Q}^{1}$ and mesh $\mathscr{W}<1 / 2 n$ while moreover $f_{n-1}(W)$ refines $\mathcal{V}^{1-1}$ and mesh $f_{n-1}(\mho) \leqslant 1 / 2 n$. For each $W \in \mho$ let $U(W)$ be the unique element of $\mathscr{Q}_{n-1}$ that contains $W$. By Lemma 2.1 for each $W \in \mathscr{W}$ there is a clopen $E(W) \subset Y_{0}$ so that

$(\alpha) \operatorname{diam}(E(W)) \leqslant \operatorname{diam}(W)+1 / 2 n$;

( $\beta) E(W) \subset U(W)$;

$(\gamma)$ if $W_{0} \neq W$, then $E\left(W_{0}\right) \cap E\left(W_{1}\right)=\varnothing$;

$(\delta)$ for each $U \in \mathscr{Q}_{n-1}: U-\cup\{E(W): E(W) \subset U\} \neq \varnothing$.

In addition, also by Lemma 2.1 , for each $W \in W$ there is a clopen $F(W) \subset Y$, so that

$\left(\alpha^{\prime}\right) \operatorname{diam}(F(W)) \leqslant \operatorname{diam}\left(f_{n-1}[W]\right)+1 / 2 n ;$

$\left(\beta^{\prime}\right) F(W) \subset V(U(W))$ and $F(W) \cap X_{t_{n-1}}^{1}=f_{n-1}[W]$;

$\left(\gamma^{\prime}\right)$ if $W_{0} \neq W_{1}$ then $F\left(W_{0}\right) \cap F\left(W_{1}\right)=\varnothing$;

$\left(\delta^{\prime}\right)$ for each $V \in \mathcal{V}_{n-1}: V-\cup\{F(W): F(W) \subset V\} \neq \varnothing$.

Fix $U \in \mathcal{Q}_{n-1}$ and consider $S=U-\cup\{E(W): E(W) \subset U\}$ and $T=V(U)-$ $\cup\{F(W): F(W) \subset V(U)\}$. Then, by $(\delta)$ and $\left(\delta^{\prime}\right), S$ and $T$ are both nonempty clopen subsets of, respectively, $Y_{0}$ and $Y_{1}$. It is clear that there is a finite disjoint clopen cover $\mathcal{E}$ of $S$ and a finite disjoint clopen cover $\mathcal{T}$ of $T$ so that $|\mathcal{E}|=|\mathcal{T}|$ while moreover mesh $\mathcal{E} \leqslant 1 / n$ and mesh $\mathcal{T} \leqslant 1 / n$. Let $V: \mathcal{E} \rightarrow \mathcal{T}$ be an arbitrary one-to-one function. If we do this for every $U \in \mathcal{Q}$ and $V(U) \in \mathcal{V}$ then the so-defined clopen sets together with the sets $\{E(W): W \in \mathscr{W}\}$ and $\{F(W)$ : $W \in \mathcal{W}\}$ form disjoint finite clopen covers of, respectively, $Y_{0}$ and $Y_{1}$. These covers are the required covers $\mathscr{Q}_{n}$ and $\mathcal{V}_{n}$. Clearly both $\mathcal{U}_{n}$ and $\mathcal{V}_{n}$ are finite and mesh $\mathscr{Q}_{n} \leqslant 1 / n$ and mesh $\mathscr{V}_{n} \leqslant 1 / n$. If we define $V(E(W))=F(W)$ then we have associated to every $U \in \mathscr{Q}_{n}$ a $V(U) \in \mathcal{V}_{n}$. Let $s_{n}$ be the first integer $k$ greater than $s_{n-1}$ for which $X_{k}^{0}$ intersects all elements of $\mathscr{U}_{n}$. Similarly, let $t_{n}$ be the first integer $l$ greater than $t_{n-1}$ for which $X_{l}^{1}$ intersects all elements of $\mathfrak{V}_{n}$. For each $U \in \mathscr{Q}_{n}$ let $g_{U}=f_{n-1} \mid U$ ( $g_{U}$ might be the empty function!). By the Homeomorphism Extension Theorem 3.1, for each $U \in \mathcal{Q}_{n}$ there is a homeomorphism $h_{U}$ : $U \cap X_{s_{n}}^{0} \rightarrow V(U) \cap X_{t_{n}}^{1}$ extending $g_{U}$. Define $f_{n}$ by

$$
f_{n}(x)=h_{U}(x) \quad\left(x \in U \in \mathcal{Q}_{n}\right) .
$$


A straightforward check shows that our inductive hypotheses are satisfied.

Now define $f: Y_{0} \rightarrow Y_{1}$ by $f(x)=f_{n}(x)\left(x \in X_{s_{n}}^{9}\right.$. It is clear that $f$ is well defined, onto, and one-to-one. We claim that $f$ is a homeomorphism.

Fact $1 . f$ is continuous.

Let $x_{i} \in Y_{0}(i \in \mathbf{N})$ be a sequence converging to $x \in Y_{0}$. Suppose that $f\left(x_{i}\right)$ does not converge to $f(x)$. Then there is an $n \in N$ and a $V \in \mathscr{V}_{n}$ containing $f(x)$ but not containing infinitely many of the $f\left(x_{i}\right)$ 's. Without loss of generality $f\left(x_{i}\right) \notin V$ for all $i \in \mathbf{N}$. We may assume that $x \in X_{s_{n}}^{0}$. Let $U$ be the unique element of $\mathscr{Q}_{n}$ for which $V(U)=V$. Notice that $x \in U$. Choose $i \in \mathbf{N}$ so that $x_{i} \in U$. Let $x_{i} \in X_{s_{m}}^{0}$ for $n<m$. In addition, let $U^{\prime} \in \mathcal{U}_{m}$ be the unique element of $\mathcal{U}_{m}$ containing $x_{i}$. Then $U^{\prime} \subset U$ and hence, by (g), $V\left(U^{\prime}\right) \subset V(U)$. Also, by (f), $f\left(x_{i}\right)=f_{m}\left(x_{i}\right) \in V\left(U^{\prime}\right)$. Since $V\left(U^{\prime}\right) \subset V(U)=V$, this is a contradiction.

Fact 2. $f^{-1}$ is continuous.

This can be proved in the same way.

We conclude that $f$ is a homeomorphism and the only remaining thing left to prove is that $Y_{0}$ is strongly homogeneous. It is clear that whenever $C$ is a nonempty clopen subset of $Y_{0}, C \in \mathrm{CAP}(X)$. Hence, by the foregoing, $C \approx Y_{0}$.

4.2. REMARK. Notice that in the proof of Theorem 4.1 we have not only used that $X$ satisfies the Homeomorphism Extension Theorem 3.1 but also, in an essential way, that $X$ is strongly homogeneous.

4.3. Remark. Eric van Douwen has observed that if $X$ is the topological sum of $\mathbf{Q} \times \mathbf{C}$ and $\mathbf{Q}$ then $\operatorname{CAP}(X)$ contains at least two spaces, namely, $X$ and $\mathbf{Q} \times \mathbf{C}$ (the proof that $\mathbf{Q} \times \mathbf{C} \in \operatorname{CAP}(X)$ uses Theorem 5.1 below). It is interesting that although $X$ is not homogeneous, $\operatorname{CAP}(X)$ contains a homogeneous space. It is unknown whether there is a homogeneous zero-dimensional space $U$ for which $\operatorname{CAP}(Y)$ contains at least two spaces.

5. Applications. In this section we give some applications of Theorem 4.1.

Since $\mathbf{Q}$ is countable we can list $\mathbf{Q}$ as $\left\{q_{n}: n \in \mathbf{N}\right\}$. It is trivial to construct a sequence $\left\{F_{n}: n \in \mathbf{N}\right\}$ of compact subspaces of $\mathbf{Q}$ so that

(a) $F_{1}=\left\{q_{1}\right\}$ and $q_{n} \in F_{n}$;

(b) $F_{n} \subset F_{n+1}$;

(c) $F_{n+1}-F_{n}$ is countable, discrete, and dense in $F_{n+1}$.

In the sequel we will make use of this sequence.

5.1. THEOREM. Let $X$ be a strongly homogeneous space containing a nowhere dense closed copy of itself. Then $\mathbf{Q} \times X \in \operatorname{CAP}(X)$.

Proof. Let us first assume that $X$ is compact. Then $X$ is homeomorphic to the Cantor set $\mathbf{C}$. Since $\mathbf{C} \times K \approx \mathbf{C}$ for each compact zero-dimensional space $K$, using the sequence $\left\{F_{n}: n \in \mathbf{N}\right\}$ described above one readily sees that $\mathbf{Q} \times X \in$ $\operatorname{CAP}(X)$.

Now assume that $X$ is not compact. First notice that this implies that $X \approx \mathbf{N} \times$ $X$. We claim that $X \approx F_{n} \times X$ for each $n \in \mathbf{N}$, where the $F_{n}$ 's are defined as above. If $n=1$ then there is nothing to prove, so assume that $X \approx F_{i} \times X$ for each $i<n$. We will show that $X \approx F_{n+1} \times X$. Since $F_{n+1}-F_{n}$ is a countable dense discrete 
subspace of $F_{n+1}$ and since $\mathbf{N} \times X \approx X$ and since $X$ contains a nowhere dense closed copy of itself, by Theorem 3.3 it follows that $F_{n+1} \times X \approx X$.

This obviously implies that $\mathbf{Q} \times X \in \operatorname{CAP}(X)$.

Notice that the above theorem gives us a topological characterization of $\mathbf{Q} \times X$.

Let $\mathcal{P}$ be a topological property. As usual, we call a space $X$ nowhere $\mathcal{P}$ provided that no point of $X$ has a neighborhood satisfying $\mathscr{P}$. A space is called $\sigma-\mathcal{P}$ whenever it is the union of countably many closed subspaces satisfying $\mathcal{P}$.

5.2. Corollary (AleXANDroff AND URysohn [1]). All nowhere locally compact, $\sigma$-compact, nowhere countable and zero-dimensional spaces are homeomorphic to $\mathbf{Q} \times \mathbf{C}$.

Proof. Let $X$ be a nowhere locally compact, $\sigma$-compact, nowhere countable and zero-dimensional space.

Fact 1. Each nonempty open subset $U$ of $X$ contains a Cantor set.

Let $C \subset U$ be nonempty and clopen. The $\sigma$-compactness of $X$ implies that $C$ is $\sigma$-compact and since $C$ is not countable, it must contain an uncountable compact space. Since each uncountable compact space contains a Cantor set, it follows that $U$ contains a Cantor set.

Fact 2. If $K \subset X$ is compact, then there is a Cantor set $E \subset X$ so that $K \subset E$ and $K$ is nowhere dense in $E$.

As in the proof of the Homeomorphism Extension Theorem 3.1 we can find a disjoint clopen cover $\mathscr{D}$ of $X-K$ so that for each $D \in \mathscr{D}$ we have that $\operatorname{diam}(D)$ $<d(D, K)$ while moreover each neighborhood of $K$ contains all but finitely many $D \in \mathscr{D}$. For each $D \in \mathscr{D}$ let $F(D)$ be a Cantor set contained in $D$ (Fact 1). Put $E=\cup_{D \in \mathscr{D}} F(D) \cup K$. A straightforward check shows that $E \approx \mathbf{C}$ while moreover $K$ is nowhere dense in $E$.

Let $X=\cup_{n=1}^{\infty} K_{n}$, where each $K_{n}$ is compact. Since $X$ is nowhere locally compact each compact subspace of $X$ is nowhere dense. By applying Fact 2 one can easily find a sequence of Cantor sets $\left\{E_{n}: n \in \mathbf{N}\right\}$ so that $K_{n} \subset E_{n} \subset E_{n+1}$ and $E_{n}$ is nowhere dense in $E_{n+1}$. Hence $X \in \operatorname{CAP}(C)$. Applying Theorem 5.1 yields $X \approx \mathbf{Q} \times \mathbf{C}$.

Let us notice that Theorem 5.1 gives us an elegant characterization of $\mathbf{Q} \times \mathbf{P}$. In view of Corollary 5.2 one would like to get a characterization of $\mathbf{Q} \times \mathbf{P}$ in more classical terms. One would be tempted to conjecture that all nowhere locally complete, $\sigma$-complete, nowhere countable and zero-dimensional spaces are homeomorphic to $\mathbf{Q} \times \mathbf{P}$. This is not true however since the Baire category theorem implies that $\mathbf{Q} \times \mathbf{P}$ and $\mathbf{Q} \times \mathbf{C}$ are not homeomorphic. We do however have the following characterization of $\mathbf{Q} \times \mathbf{P}$.

5.3. Corollary. Let $X$ be a zero-dimensional $\sigma$-complete space which in addition is nowhere complete and nowhere $\sigma$-compact. Then $X$ is homeomorphic to $\mathbf{Q} \times \mathbf{P}$.

Proof. The pattern of proof is the same as in the proof of Corollary 5.2.

Fact 1. Each nonempty clopen subspace $C$ of $X$ contains a closed copy of $\mathbf{P}$.

Since $X$ is $\sigma$-complete, so is $C$. Let $C=\cup_{n-1}^{\infty} C_{n}$, where each $C_{n}$ is a closed and topologically complete subspace of $C$. Since $C$ is not $\sigma$-compact, one of the $C_{n}$ 's is 
not $\sigma$-compact, say $C_{k}$. Let $\delta$ be the family of all clopen (in $C_{k}$ ) $\sigma$-compact subspaces of $C_{k}$. Since $C_{k}$ is not $\sigma$-compact, $\cup \mathcal{E} \neq C_{k}$. Put $P=C_{k}-\cup \mathcal{E}$. We claim that $P$ is nowhere locally compact. To the contrary, assume that $F \subset P$ is a nonempty clopen (in $P$ ) compact subspace. Let $F^{\prime} \subset C_{k}$ be clopen so that $F^{\prime} \cap P$ $=F$. There is a countable subfamily $\mathcal{E}^{\prime} \subset \mathcal{E}$ so that $\cup \mathcal{E}^{\prime}=F^{\prime}-P$. Since $F$ is compact, it follows that $F^{\prime}$ is $\sigma$-compact, a contradiction since $F^{\prime} \cap P \neq \varnothing$. Also, $P$ is topologically complete, since $P$ is a closed subspace of $C_{k}$. We conclude that $P \approx \mathbf{P}[1]$.

Fact 2. If $K \subset X$ is closed and (topologically) complete, then there is a closed copy $E$ of $P$ in $X$ so that $K \subset E$ and $K$ is nowhere dense in $E$.

As in the proof of the Homeomorphism Extension Theorem 3.1 we can find a disjoint clopen cover $\mathscr{D}$ of $X-K$ so that for each $D \in \mathscr{D}$ we have that $\operatorname{diam}(D)$ $<d(D, K)$. For each $D \in \mathscr{D}$ let $F(D) \subset D$ be a closed copy of $\mathbf{P}$ and put $E=\cup_{D \in \mathscr{D}} F(D) \cup K$. It is clear that $E$ is closed in $X$ and that $K$ is nowhere dense in $E$. It remains to show that $E$ is complete [1]. For convenience put $P=\cup_{D \in \mathscr{D}} F(D)$. Notice that $P \approx \mathbf{P}$ and that $P$ is dense and open in $E$. Let $\tilde{E}$ be a compactification of $E$. Then $\tilde{E}-P$ is $\sigma$-compact, since $P$ is complete. Hence $\tilde{E}-(P \cup \bar{K})$ is also $\sigma$-compact. Since $K$ is complete the remainder $\bar{K}-K$ is $\sigma$-compact. We conclude that $\tilde{E}-E$ is $\sigma$-compact. Consequently, $E$ is complete [6, 4.3.26].

The proof can now be completed in precisely the same way as in the proof of Corollary 5.2.

5.4. REMARK. In an earlier version of this paper we proved that if $X$ is a zero-dimensional nowhere complete, $\sigma$-complete space with the additional property that each nonempty clopen subset of $X$ contains a closed copy of $\mathbf{P}$, then $X$ is homeomorphic to $\mathbf{Q} \times \mathbf{P}$. Eric van Douwen informed us that the condition that each nonempty clopen subset of $X$ contains a closed copy of $\mathbf{P}$ can be replaced by nowhere $\sigma$-compactness, and hence that the characterization of $\mathbf{Q} \times \mathbf{P}$ stated here as Corollary 5.3 holds. I am indebted to Eric van Douwen for pointing that out to me since Corollary 5.3 is obviously a more interesting characterization of $\mathbf{Q} \times \mathbf{P}$ than the one stated above, since it does not mention $\mathbf{P}$.

6. Remarks. The results derived in this paper suggest many questions. The most important unsettled question probably is to find a topological characterization of $\mathbf{Q}^{\infty}$, i.e., the topological product of countably many copies of $\mathbf{Q}$. The only information we have is that $\mathbf{Q}^{\infty} \in \operatorname{CAP}\left(\mathbf{Q}^{\infty}\right)$ (which is trivial) and that $\mathbf{Q}^{\infty}$ is not $\sigma$-complete [7].

The following question is suggested by results in infinite dimensional topology.

6.1. Question. Are the following two statements equivalent?

(a) $X$ is embeddable as a closed subspace of $\mathbf{Q}^{\infty}$;

(b) $X \times \mathbf{Q}^{\infty} \approx \mathbf{Q}^{\infty}$.

Let us finally notice that in his interesting paper [9], Gutek showed that each homeomorphism $\Phi$ between nowhere dense closed subsets of the Cantor set $\mathbf{C}$ can be extended to an autohomeomorphism $\bar{\Phi}: \mathbf{C} \rightarrow \mathbf{C}$ with a dense orbit (i.e. there is an $x \in \mathbf{C}$ so that $\left\{\bar{\Phi}^{n}(x): n \in \mathbf{N}\right\}$ is dense in $\left.\mathbf{C}\right)$. This suggests the following question. 
6.2. Question. Let $X$ be a strongly homogeneous zero-dimensional space and let $f$ : $F \rightarrow G$ be a homeomorphism between closed nowhere dense subsets of $X$. Can $f$ be extended to an autohomeomorphism $\bar{f}$ of $X$ with a dense orbit?

\section{REFERENCES}

1. P. Alexandroff and P. Urysohn, Über nulldimensionale Punktmengen, Math. Ann. 98 (1928), 89-106.

2. R. D. Anderson, On topological infinite deficiency, Michigan Math. J. 14 (1967), 365-383.

3. L. E. J. Brouwer, On the structure of perfect sets of points, Proc. Akad. Amsterdam 12 (1910), 785-794.

4. E. K. van Douwen, A measure that knows which sets are homeomorphic, Top. Structures 2 , Mathematical Centre Tracts 115 (1979), 67-71.

5. _ Destroying nowhere dense copies or group structure in strongly homogeneous zero-dimensional spaces (to appear).

6. R. Engelking, General topology, Polish Scientific Publishers, Warszawa, 1977.

7. R. Engelking, W. Holsztyński and R. Sikorski, Some examples of Borel sets, Colloq. Math. 15 (1966), 271-274.

8. J. de Groot and R. H. McDowell, Extensions of mappings on metric spaces, Fund. Math. 48 (1960), 251-263.

9. A. Gutek, On extending homeomorphisms on the Cantor set, Top. Structures 2, Mathematical Centre Tracts 115 (1979), 105-116.

10. P. R. Halmos, Lectures on Boolean algebras, Princeton Univ. Press, Princeton, N. J., 1963.

11. , Permutations of sequences and the Schröder-Bernstein theorem, Proc. Amer. Math. Soc. 19 (1968), 509-510.

12. B. Knaster and M. Reichbach, Notion d'homogénéité et prolongements des homéomorphies, Fund. Math. 40 (1953), 180-193.

13. J. von Neumann, Characterisierung des Spektrums eines Integral-operators, Hermann, Paris, 1935.

14. J. Pollard, On extending homeomorphisms on zero-dimensional spaces, Fund. Math. 67 (1970), 39-48.

15. W. Sierpiński, Sur une propriété topologique des ensembles denombrables denses en soi, Fund. Math. 1 (1920), 11-16.

16. J. A. Yorke, Permutations and two sequences with the same cluster set, Proc. Amer. Math. Soc. 20 (1969), 606.

Department of Mathematics, Louisiana State Universtty, Baton Rouge, Louisiana 70803

Current address: Subfaculteit Wiskunde, Vrije Universiteit, De Boelelaan 1081, Amsterdam, The Netherlands 